Research Article

\title{
On the Suppression Band and Bandgap of Planar Electromagnetic Bandgap Structures
}

\author{
Baharak Mohajer-Iravani and Omar M. Ramahi \\ Electrical and Computer Engineering Department, University of Waterloo, Waterloo, ON, Canada \\ Correspondence should be addressed to Omar M. Ramahi; oramahi@uwaterloo.ca
}

Received 17 June 2013; Revised 16 September 2013; Accepted 19 September 2013; Published 2 January 2014

Academic Editor: Stefano Selleri

Copyright (C) 2014 B. Mohajer-Iravani and O. M. Ramahi. This is an open access article distributed under the Creative Commons Attribution License, which permits unrestricted use, distribution, and reproduction in any medium, provided the original work is properly cited.

\begin{abstract}
Electromagnetic bandgap structures are considered a viable solution for the problem of switching noise in printed circuit boards and packages. Less attention, however, has been given to whether or not the introduction of EBGs affects the EMI potential of the circuit to couple unwanted energy to neighboring layers or interconnects. In this paper, we show that the bandgap of EBG structures, as generated using the Brillouin diagram, does not necessarily correspond to the suppression bandwidth typically generated using $S$ parameters. We show that the reactive near fields radiating from openings within the EBG layers can be substantial and are present in the entire frequency band including propagating and nonpropagating mode regions. These fields decay fast with distance; however, they can couple significant energy to adjacent layers and to signal lines. The findings are validated using full-wave three-dimensional numerical simulation. Based on this work, design guidelines for EBG structures can be drawn to insure not only suppression of switching noise but also minimization of EMI and insuring signal integrity.
\end{abstract}

\section{Introduction}

The interests to implement electromagnetic bandgap (EBG) structures as a viable solution to reduce electromagnetic interference (EMI) and control switching noise are increasing. However, the size of EBG structures and space restriction on packages pose a challenge. Fortunately, new advanced materials recently produced when combined with EBG structures present possible solutions to the persisting bottlenecks. Amongst newly developed materials are those with very high relative permittivity (above 100). In industrial circles, these materials are typically referred to as high- $k$ materials. In fact, the use of high- $k$ materials in multilayer technologies including printed circuit board (PCB) and low temperature cofired ceramic (LTCC) is becoming popular [1].

In recent work, miniaturized planar EBG structures were designed using high $-k$ materials and were introduced as a highly effective mechanism for suppressing switching noise in high speed integrated printed circuit boards and packages [2-4]. The potential and advantages of adapting these new designs were discussed and validated using numerical full-wave three-dimensional electromagnetic simulators.
The assessment of attenuation loss between two ports within a board/package parallel layers showed that the EBG patterning of the layer creates a very wide suppression band. However, later studies in [5] showed that the extracted dispersion diagrams for the unit cell of infinite one-dimensional EBG array based on an equivalent circuit model and numerical simulation demonstrated the presence of propagating modes within the suppression band. Such interesting findings raise important questions. What happens to the energy of these modes and why the magnitude of insertion loss when calculated or measured between two ports does not show such modes?

In [6], we presented preliminary results that discussed discrepancies between an effective suppression band, as obtained from $S$-parameters, and a bandgap, as obtained from Brillouin diagram for two-dimensional (2D) EBG structures. In [6], we discussed the potential of radiation from EBG structures at frequencies corresponding to those of higher order slow modes existing in the suppression band by assuming negligible material losses in the EBG patterned parallel planes. In [7], near-field measurements for FR4-based planar EBG structures, which are excited through a source driving 
propagating modes between EBG planes, showed radiations to surrounding environment. The work in [7], however, did not present any analysis of the far field radiation. Radiation from EBGs, which are bandstop filters in their bandgaps, is naturally undesired. Such radiation can be detrimental to the surrounding devices. Therefore, a thorough study is needed to predict the frequency band where radiation takes place and to provide a validation for such prediction. Such study can answer the question if an effective suppression band or bandgap is sufficient for a particular application.

In this paper, we show that material losses are not negligible as was assumed in [6]. Based on this new understanding, the differences between a suppression band and the EBG bandgap are addressed. We analyze the power loss and power transfer between two points in the presence of EBG structures. This analysis provides a better understanding of the mechanism of radiation from EBG patterned parallel planes within and outside the bandgap regions. We conclude with preliminary design guidelines for EBG structures that achieves not only switching noise suppression but also reduced emissions and good signal integrity.

We emphasize that this study is concerned with the electromagnetic noise produced by simultaneous switching noise which primarily affects the power distribution network. Switching noise is produced by fast switching CMOS devices. This switching is typically and effectively modeled as a current source between two reference planes (see [8-12] and references therein). Different types of interference arise due to signal routing above reference planes that might be EBG patterned as in [9]. While we acknowledge the importance of understanding the radiation due to the placement of such traces, this study is concerned with interference and radiation caused by switching noise.

\section{Characterization of EBG Structure}

As a test case for this study, we consider a planar EBG structure consisting of two planar metallic parallel plates. On the first layer, the EBG patches connected with meander lines are etched while the second layer is plain solid. A very thin layer of high- $k$ substrate is sandwiched between the two metallic layers. The metal layers are assumed to be perfect electric conductors (PEC) as metallic loss is negligible over the frequency range used in this study. The top view of the $2 \mathrm{D}$ array of periodic planar EBG structures is shown in Figure 1(a). This structure was introduced in [2] and incorporated in package design in $[3,4]$. We emphasize that this structure was selected merely as an example of a planar EBG power plane as the analysis presented here applies equally to other planar structures.

To characterize the behaviour of the wave as it propagates through the structure, we assume a propagation constant of $\vec{k}=k_{x} \hat{x}+k_{y} \hat{y}$. The computational domain and the boundary setup for extracting the dispersion diagram for the EBG unit cell are shown in Figure 1(b) $[13,14]$. Periodic boundary condition (PBC) mimics the periodicity in the infinite 2D array and an absorbing boundary condition or a perfectly matched layer (PML) on the top wall resembles the free space termination. The full-wave numerical simulator Ansoft HFSS [15] is used to analyse the 2D propagating and nonpropagating modes on the irreducible Brillouin diagram [16] which is shown in Figure 1(c). The top view of this unit cell is shown in Figure 1(d).

For comparison purposes, we follow our earlier work on the same EBG sample as presented in $[3,4]$. The EBG sample is defined by the following design parameters: square patch width $P_{W}=1.6 \mathrm{~mm}$, meander-arm length $M_{L}=1.6 \mathrm{~mm}$, meander-gap length $M_{G}=0.02 \mathrm{~mm}$, meander-strip width $M_{W}=$ $0.02 \mathrm{~mm}$, dielectric thickness $h=114.3 \mu \mathrm{m}$, and dielectric relative permittivity $\varepsilon_{r}=140$ and loss tangent $\tan \delta=0.0015$.

Figure 2 shows the dispersion data points of the first four propagating modes extracted numerically for this structure. The dispersion curves of periodic structures show several extrema or points of zero-group velocity along the propagating bands, particularly, at the center or edge of Brillouin zone. The dispersion diagram in Figure 2 also shows the bandgap frequency regions confined by propagating modes at the bottom and the top frequency edges, at the extrema points. This means that the propagating modes change to slow modes at the bandgap edges. This can be analysed by careful consideration of the group velocity defined by $v_{g}^{-1}=\partial \beta / \partial \omega$.

Figure 3 shows the group velocity diagrams corresponding to the dispersion curves in Figure 2 for the regions of the irreducible Brillouin zone, that is, $\Gamma-X, X-M$, and $M-\Gamma$. We note here that the group velocity graphs were obtained by using polynomials that approximate the data generated for the dispersion diagrams and then performing the differentiation as stipulated by the definition of the group velocity. The fitted polynomials, which estimate the dispersion relation in each region of the irreducible Brillouin zone but not very close to its center or edges, are also plotted in Figure 2. The light line plotted in each region defines the wave numbers for the plane wave travelling through the host medium. Notice that the group velocity of the propagating modes that are close to the edges of bandgap is more than 10 times slower than the velocity of light in the dielectric medium which is equal to $25.36 \mathrm{Mm} / \mathrm{s}$. Moreover, each of the higher order propagating modes (including the second, the third, and the forth modes which are shown in the figure) over the frequency range of propagation is a slow wave.

The dispersion diagrams or the group velocity diagrams generated for the EBG sample show that the first bandgap is located between $1.9 \mathrm{GHz}$ and $4 \mathrm{GHz}$ and the second bandgap falls between $4.5 \mathrm{GHz}$ and $5.4 \mathrm{GHz}$. However, the $20-\mathrm{dB}$ suppression band for this sample, which is extracted from the transferred power between two ports (as represented by the $S_{21}$ parameter) using the setup shown in Figure 4, extends from $2.77 \mathrm{GHz}$ to $10.67 \mathrm{GHz}$. Figure 5 shows the magnitude of $S_{21}$ for the EBG patterned parallel planes and the corresponding solid parallel planes as a reference case. Clearly, the frequency range for the suppression band is different from the bandgap frequency ranges. Also, the suppression band consists of few bandgaps and few higher order propagatingmodes which are partially shown in Figure 2.

The following reasons are provided to explain the difference between the locations of the two extracted bands. First, 


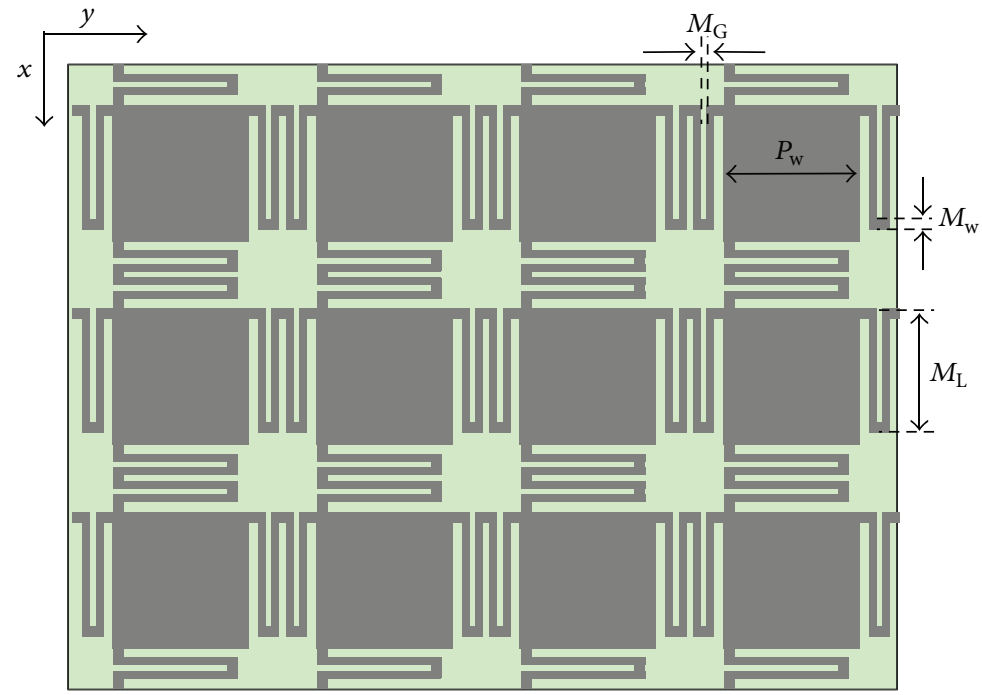

(a)

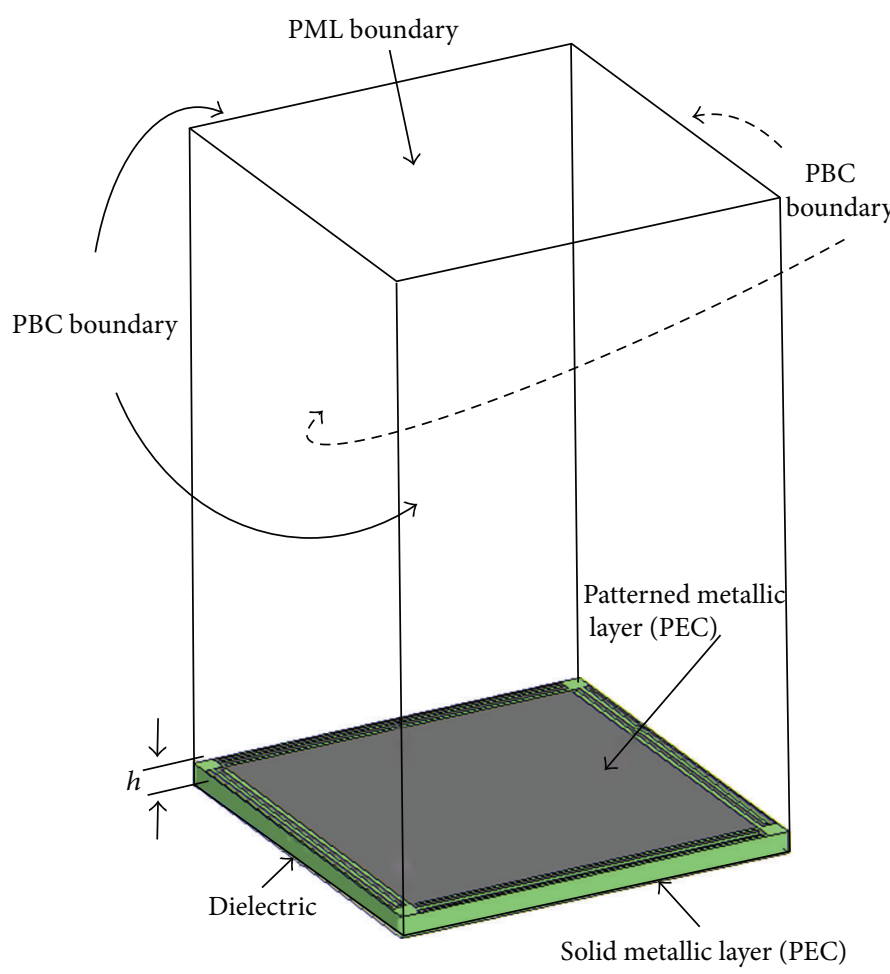

(b)

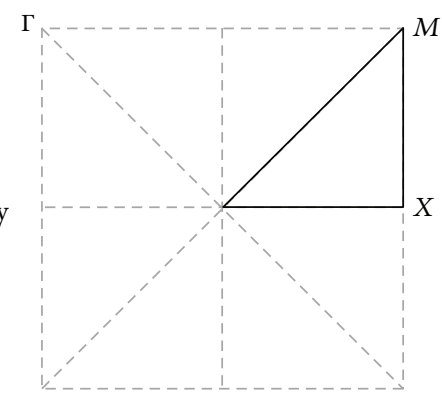

(c)

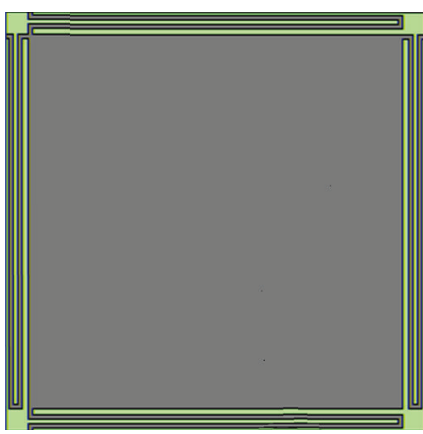

(d)

FIgURE 1: (a) Top view of 2D periodic planar EBG structures made of patch and two-turn meander lines. (b) Computational setup used to extract the dispersion diagram of the EBG-unit cell in infinite array. The appropriate boundaries are shown in the setup. (c) Irreducible Brillouin zone triangle. (d) Top view of the unit cell.

the dispersion diagram is extracted for an ideal case where the array of periodic EBG structures is infinite, whereas, the suppression band is extracted for a finite number of EBG structures. In the numerical measurement setup provided in Figure 4, two ports are separated by only 4 unit cells of EBGs. One can also expect radiation to the surrounding environment and multiple reflections due to the discontinuity in the structure. Second, within the bandgap, evanescent modes exist with limited attenuations per unit-cell length. The infinite number of EBG cells in the infinite array contributes to infinite-attenuation level in each bandgap. In other words, it is possible not to obtain even limited suppression level (say of $20 \mathrm{~dB}$ ) by applying only a few numbers of EBG cells. Third, a bandgap implies the absence of propagating modes, whereas, a suppression band does not necessarily imply the absence of propagating modes. More specifically, 


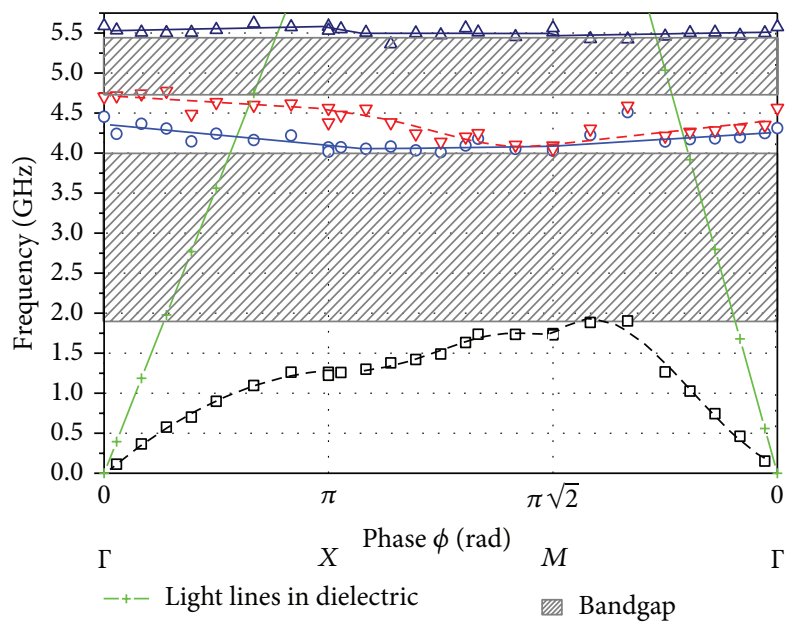

Propagating-mode-data sets Polynomials fit to the mode-data sets

$\begin{array}{ll}\square \text { 1st } & --1 \text { st } \\ \circ \text { 2nd } & -2 \text { nd } \\ \nabla \text { 3rd } & --3 \text { rd } \\ \Delta 4 \text { th } & -4 \text { th }\end{array}$

FIGURE 2: Dispersion diagram of the planar EBG structure shown in Figure 1 and light lines in dielectric. Four sets of data points showing the first four propagating modes are extracted numerically using HFSS. The curves fitted to these sets of data are plotted in the diagram. The design parameters of the EBG are: $P_{W}=1.6 \mathrm{~mm}, M_{W}=$ $0.02 \mathrm{~mm}, M_{L}=1.6 \mathrm{~mm}, M_{G}=0.02 \mathrm{~mm}, h=114.3 \mu \mathrm{m}, \varepsilon_{r}=140$, and $\tan \delta=0.0015$.

a suppression band implies the absence of power transfer. In other words, in the suppression band the level of transferred power between two ports which is either through evanescent modes or propagating modes is reduced due to the presence of the EBG structures.

These facts explain the difference between the start frequencies of the suppression band and the first bandgap.

\section{Power Loss Analysis}

The first explanation that comes to mind to explain the incompatibility between the suppression band and the bandgap is radiation loss through the slits (openings between EBG patches) and edges of the board. Another explanation is that higher order modes become severely attenuated after propagation in the EBG structures due to the dielectric loss. The total normalized power loss is quantified by $1-\left|S_{11}\right|^{2}-$ $\left|S_{21}\right|^{2}$, where all power quantities are normalized. The two power loss possibilities are analysed separately.

Assuming the material power losses to be negligible $P_{\mathrm{LM}} \approx 0$, then the radiation power loss, with good approximation, is computed by $1-\left|S_{11}\right|^{2}-\left|S_{21}\right|^{2}$. Our objective is to investigate the hypothesis of radiation from the openings of EBG structures to surrounding environment at frequencies compatible to higher-order propagating modes. It is possible that the slow modes, including higher order modes and modes at the edges of suppression band, increase the radiation of the overall structure. The radiation power loss is calculated for the planar EBG structures using the $S$-parameters extracted from the setup shown in Figure 4. For comparison purpose, the radiation power loss is also computed for the solid parallel planes with the same dimensions and the port locations as the EBG patterned parallel planes. Recall that solid parallel planes radiate power from the edges of the board. Such radiation is enhanced at the resonant frequencies of parallel planes.

The radiation power loss parameters of the EBG patterned parallel planes and that of the corresponding reference case are shown in Figure 6, where the 20-dB suppression band of the EBG structure corresponding to $2.77 \mathrm{GHz}-10.67 \mathrm{GHz}$, as directly obtained from Figure 5, is also marked. Comparison between the loss graphs in the effective frequency band of EBG shows that EBG patterning strongly decreases the local peaks of radiation loss except at very few highly isolated peaks where the power loss increases in comparison to the power plane with solid layers. This means that EBG patterning not only suppresses switching noise but also reduces, for most of the frequency band, emissions to the surrounding environment. Interesting and important observations are in order.

Outside the effective suppression band, especially close to the edges of this band, the radiation loss from the EBG patterned parallel planes increases. The radiation power loss diagram shows that several peaks are densely concentrated at the edges of suppression band. In addition, within the suppression band region, there are few highly isolated local peaks that have substantial amplitude. Comparing the frequency bands of these peaks with the frequency bands of the slow propagating modes in the dispersion diagram presented in Figure 2 (or the group velocity diagram given in Figure 3 ) shows strong correspondence between the two. A few of these in-band peaks are marked as slow modes in Figure 6. Of course, one can argue from the circuit point of view that patterning the package layer increases the inductance of the planes; therefore, the package layer resonates at different frequencies which are shifted to lower frequency regimes compared to the resonances of solid package. At the new resonance frequencies, the radiation potential of the board increases. In the bandgaps, the radiation power losses are negligible. This is consistent with the theory of planar bandstop filter design. Study of other examples of planar EBGs showed similar behavior [17].

Figure 7 shows the graphs of average radiated power from EBG-patterned parallel plane setup and the reference solid parallel plane setup. This is the amount of total real power exiting from the structure through the radiation boundary in the computational domain and it is computed based on the integration of Poynting vector on the radiation boundary surface [18]. The results clearly indicate that the total radiated real power for the EBG plane compared to the case with a solid plane, in the suppression band, is on the average decreased. In the bandgaps, the reduction in the radiated power is profound. Notice that the scale of power shows that in general the radiated power from the EBG structure is very small; thus, the proposition that the slow waves (at the edges of the bandgap) cause appreciable radiation is not valid. This leaves us with the conclusion that the power loss is mainly due to the material losses in the boards. This implies that the assumption of negligible material losses is not accurate, 


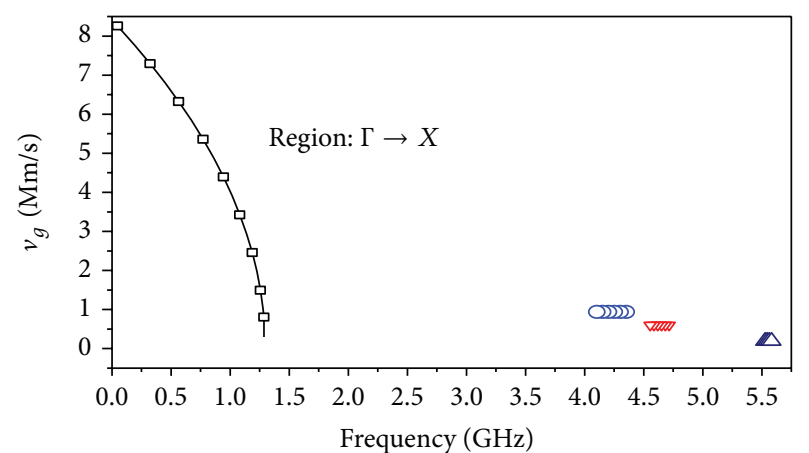

Propagating modes

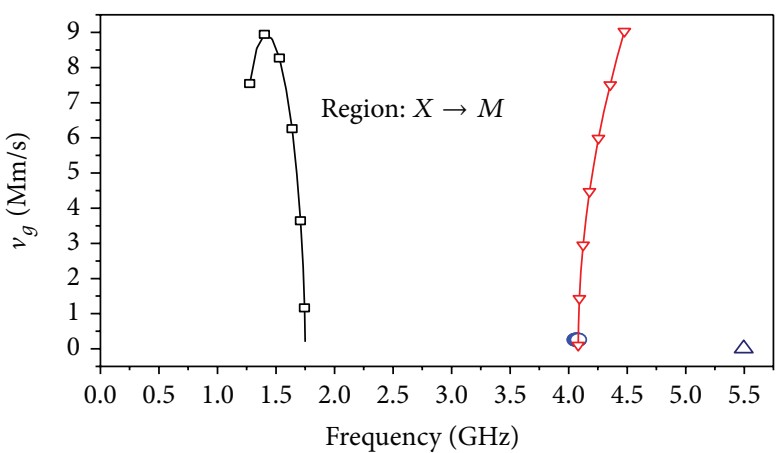

Propagating modes

(a)

(b)

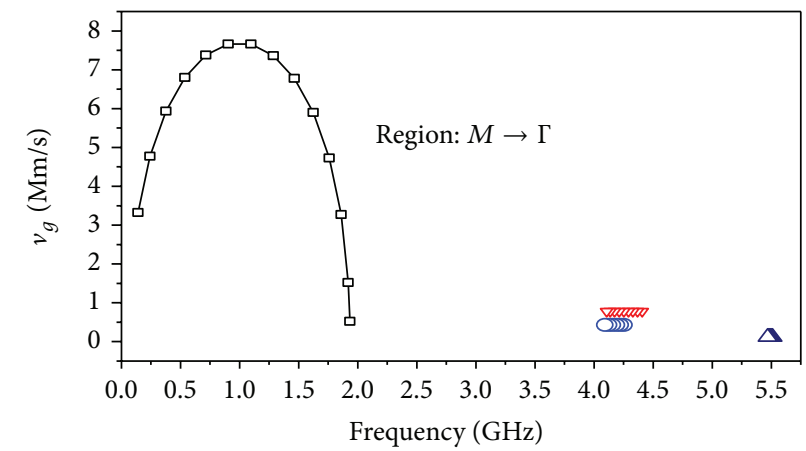

Propagating modes

$\begin{array}{ll}\rightarrow-1 \text { st } & \rightarrow \text { rd } \\ -0-2 \text { nd } & \triangle 4 \text { th }\end{array}$

(c)

FIGURE 3: Group velocity $v_{g}$ of the propagating modes of the EBG sample in irreducible Brillouin zone. The $v_{g}$ graphs are obtained from the propagating-mode-polynomial fits presented in Figure 2.

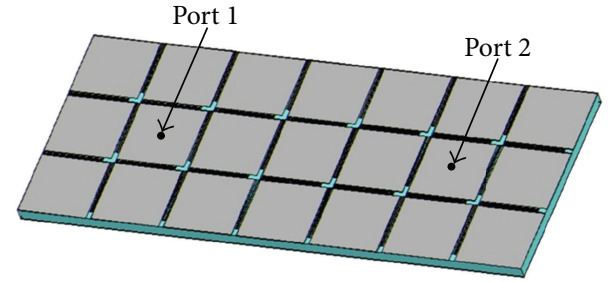

Figure 4: Setup used to extract the suppression band and the power loss parameter of EBG structures. The port locations are shown in the figure.

implying that the results presented in Figure 6, including the in-band peaks and the peaks at the edges of the suppression band, show the total power mainly lost inside the parallelplate boards.

The material or dielectric power loss inside the parallelplane setup is calculated by [18]

$$
P_{\mathrm{LM}}=\frac{\omega}{2} \int_{V} \varepsilon^{\prime \prime}|\vec{E}|^{2} d v
$$

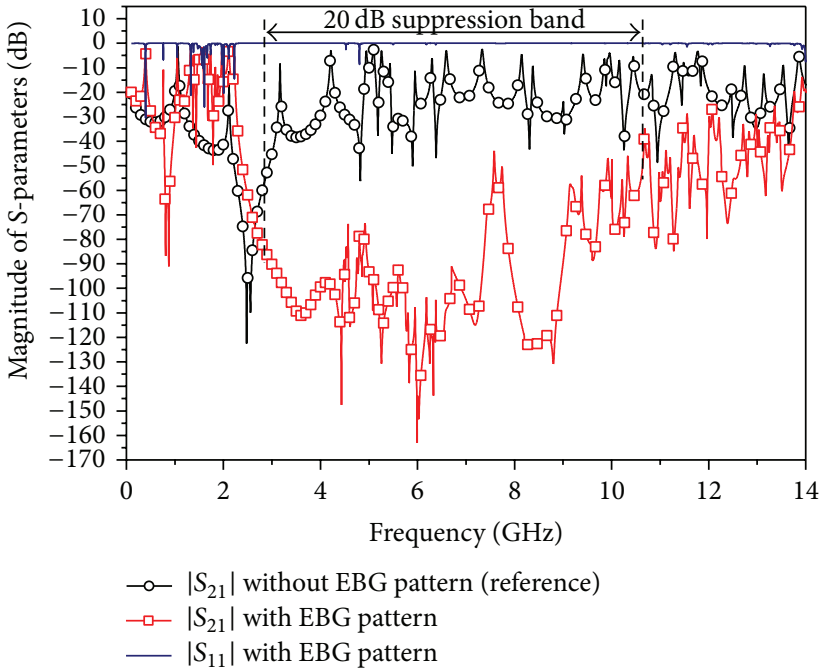

FIgURE 5: Magnitude of $S_{21}$ versus frequency for two setups: (i) the EBG-patterned parallel planes as shown in Figure 4 and (ii) the corresponding solid parallel planes as a reference. The EBG structures defined in the caption of Figure 2. 


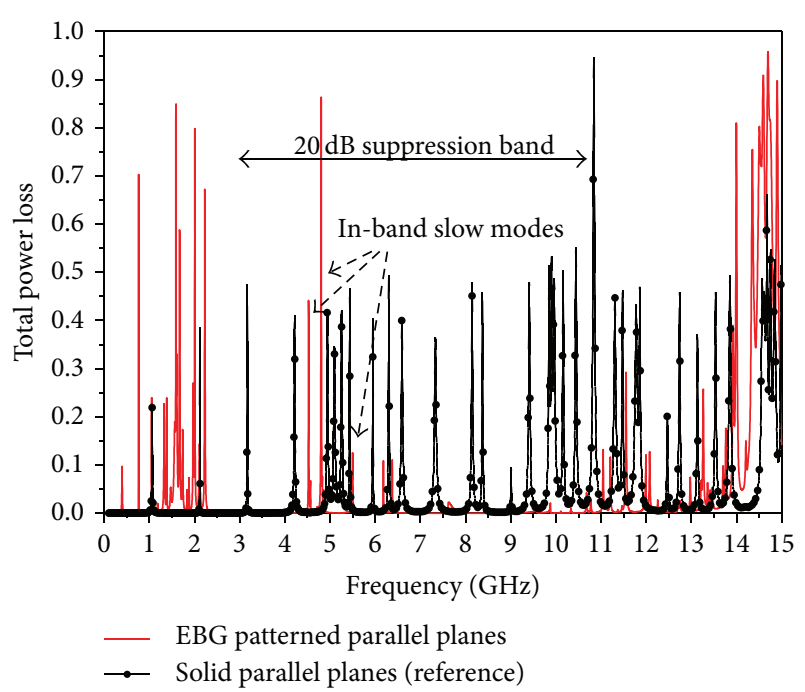

FIGURE 6: Total power loss $\left(1-\left|S_{11}\right|^{2}-\left|S_{21}\right|^{2}\right)$ versus frequency for two setups: (i) the EBG-patterned parallel planes as shown in Figure 4 and (ii) the corresponding solid parallel planes as a reference.

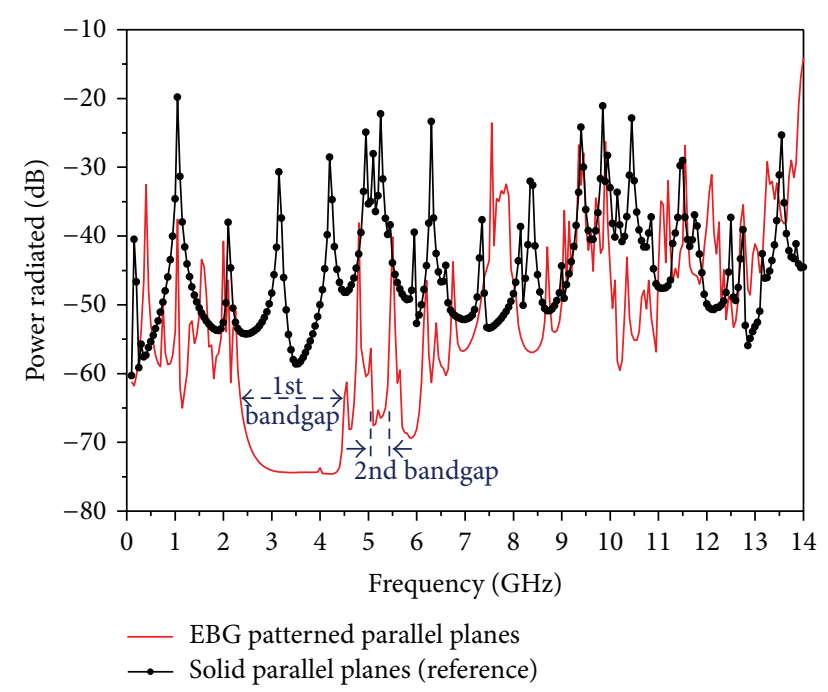

FIGURE 7: Real radiated power versus frequency from two setups defined in the caption of Figure 6.

where the imaginary part of dielectric permittivity is defined by $\varepsilon^{\prime \prime}=\varepsilon^{\prime} \tan \delta . \varepsilon^{\prime}$ is the real part of dielectric permittivity. The power loss density diagrams inside the EBG-patterned setup for three different frequencies including $2 \mathrm{GHz}$, which samples the first propagating mode region, $3.5 \mathrm{GHz}$, which samples the first bandgap, and $4.5 \mathrm{GHz}$, which samples the second propagating mode region, are shown in Figures $8(a)-8(c)$, respectively. (The mode charts were presented in Figure 2.) For field computations, only one port is excited. In our case, this active transmitting port is the right one labeled as Port 2. The other port acts as a receiver. Figure 8(a) shows that the power of first propagating mode $(2 \mathrm{GHz})$ dissipates within the entire setup due to the material losses. Based on the colour scale presented, this loss is considerable everywhere in the setup. Figure 8(b) clearly shows that, for evanescent nonpropagating modes inside the bandgap, the material power loss is localized only around the active transmitting port. Figure $8(\mathrm{c})$ shows that, for a higher-order propagating mode $(4.5 \mathrm{GHz})$ in the suppression band, the material power loss is localized around the active port mainly underneath of two sets of meander lines adjacent to this port. The power loss decreases very quick with distance from the active port.

The material power losses for the EBG-patterned parallel planes and for the solid parallel planes (while one port is used as the excitation and the other port is the receiver) are computed and shown in Figure 9. Due to the symmetry of the open lossy two-port system, the power loss inside the setup is the same independent of which port is excited. The results confirm that the material power loss is quite large specifically in the frequency regions corresponding to local peaks shown in Figure 6. Hence, at the slow propagating mode frequency regions, the power is mostly dissipated in the material.

The suppression band and results of Figure 5 showed that several of higher-order modes which fall in the suppression band (such as the second, the third, and the forth modes presented in dispersion diagrams) are not detectable in $S$ parameters calculations. However, when plotting the material power loss over the entire frequency range of interest, as shown in Figure 9, we observed that material power loss for higher-order modes is considerable. This leads us to conclude that the ports located in the middle of EBGs in the test setup do not couple enough energy to these hidden higher-order modes. Also, the coupled energy to these higher-order modes dissipates inside the structures. Hence, these higher-order modes are evanescent in the wide suppression band.

Another important consideration to keep in mind is that the dispersion diagram presents the modes that can possibly exist in the structure. However, the dispersion diagram does not necessarily mean that a source in the structures excites all of these modes. In our case study, the ports were positioned in the middle of an EBG cell, which should not be expected to excite all possible modes in the dispersion diagram. To confirm the effect of EBG arrangements around the ports on the modes propagation, we studied other test setups where the two ports were positioned off the middle of EBG unit cells (contrary to setup shown in Figure 4) [19]. It was observed that the sets of modes detected in the $S$-parameters depend on the port positions. The results of this study are not included here for brevity but a summary is as follows. It is possible to optimize the location of EBG patterns around the ports to achieve a suppression band which is much wider than a bandgap. In fact, the effective suppression band can be sufficiently wide and within this band evanescent higher-order modes and nonexcited modes can be present. This explains the results presented above where the effective suppression band is very wide and is different from the bandgap and propagating mode charts extracted via the dispersion diagram.

In the lossy-open two-port parallel-plane system, the radiation power loss is quantified by the value of $1-\left|S_{11}\right|^{2}-$ $\left|S_{21}\right|^{2}-P_{\mathrm{LM}}$. The radiation power losses calculated for the EBG patterned planes and its corresponding reference case 


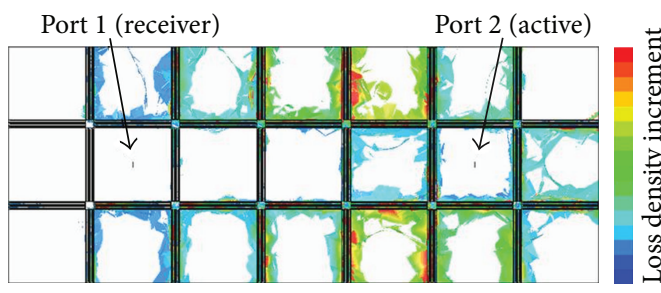

(a)

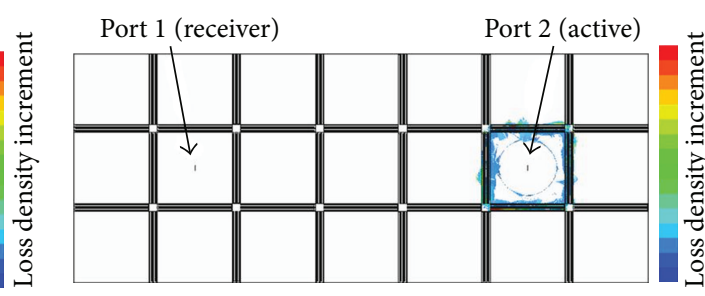

(b)

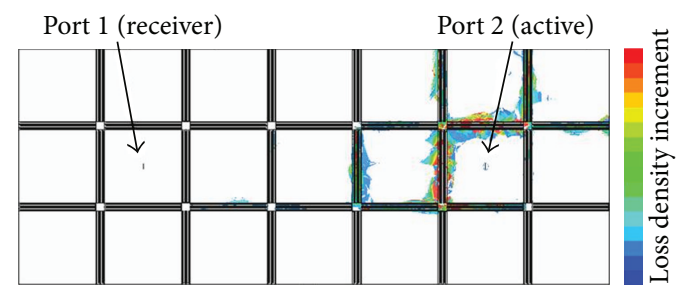

(c)

FIGURE 8: Material power loss density per volume inside the EBG-patterned parallel-plane setup: (a) at $2 \mathrm{GHz}$, (b) at $3.5 \mathrm{GHz}$, and (c) at $4.5 \mathrm{GHz}$.

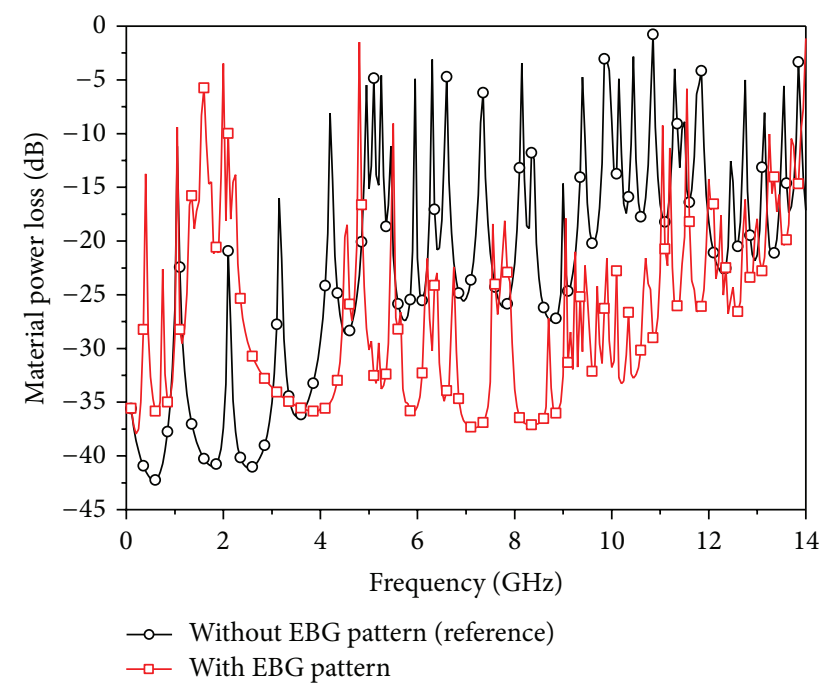

Figure 9: Material power loss $P_{\mathrm{LM}}$ versus frequency for two setups defined in the caption of Figure 6.

are plotted in Figure 10. These results, which are comprised of radiation loss data and numerical errors, confirm that the power lost through radiation is very small. Basically, the tolerance error is less than $2 \%$ in the entire frequency band except at far most edge around $14 \mathrm{GHz}$ where the error of numerical data may reach $4 \%$. The power loss is always a positive value. The negative values in the graph present the numerical errors which are inevitable in numerical simulations. The radiation power loss data corresponds to radiated real power from structures presented in Figure 7.

The radiation gains perpendicular to the board planes (at $\theta=0, \varphi=0)$ for the EBG setup and the reference setup are shown in Figure 11. The peak gains (which are not necessarily in the perpendicular direction to planes) for these setups are presented in Figure 12. The results in Figure 12 again confirm

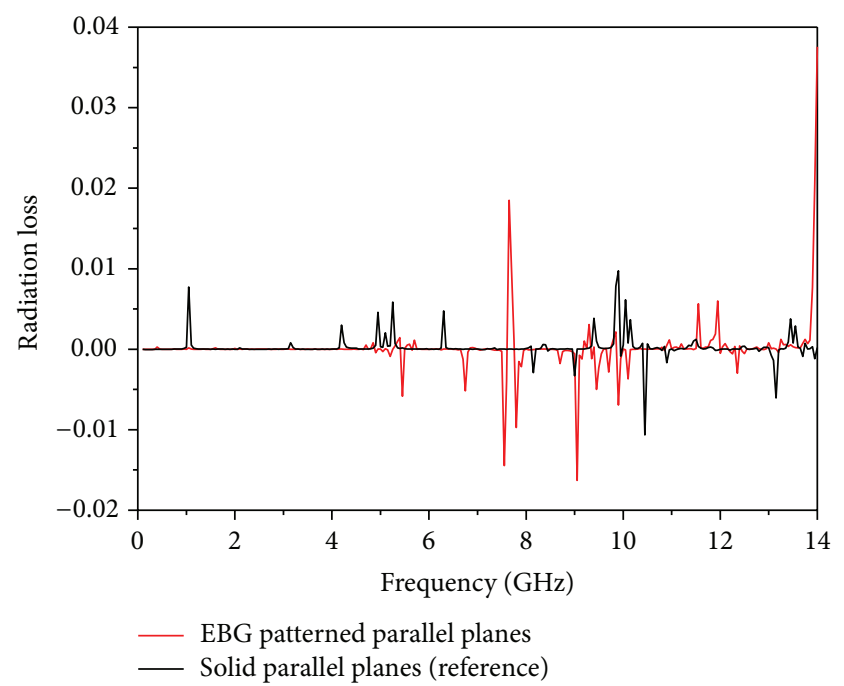

FIGURE 10: Radiation power loss $\left(1-\left|S_{11}\right|^{2}-\left|S_{21}\right|^{2}-P_{\mathrm{LM}}\right)$ versus frequency for two setups defined in the caption of Figure 6 . The material loss $P_{\mathrm{LM}}$ is presented in Figure 9.

that the radiation when using the EBG patterned plane in the suppression band compared to the radiation when using the traditional solid planes is mostly decreased in the entire frequency band.

It is important for EMI/EMC engineers who are interested in the functionality and signal integrity aspects of EBG design to consider that an effective suppression band between two ports is not necessarily equivalent to a bandgap. If there is any unexcited mode or evanescent mode in a suppression band then in the package setup, there is a likelihood that such mode may be excited through other interconnects and transmission lines (i.e., not the ports that mimic the behavior of the switching current between ground planes) that can couple energy to EBG designs. 


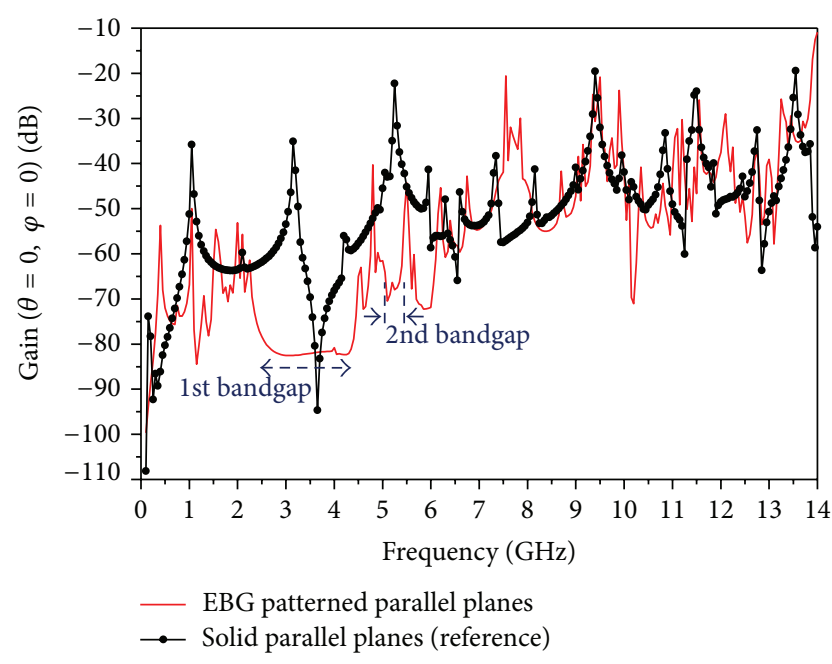

FIGURE 11: Radiation gain versus frequency from two setups defined in the caption of Figure 6 in perpendicular direction to the planes.

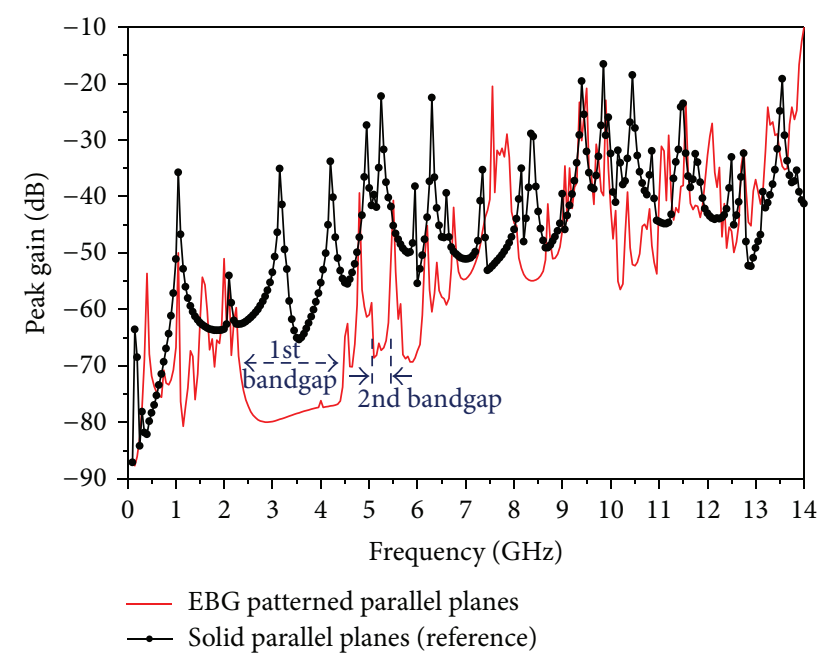

FIGURE 12: Radiation peak gain versus frequency from two setups defined in the caption of Figure 6.

\section{Reactive Power: A Source of EMI}

In previous sections, it was shown that, for the EBG power plane under study, the power loss for both propagating and evanescent modes was mostly due to material losses in the structures (dielectric loss). The radiated power was observed to be small. Hence, the fields carrying this power are not expected to be major source of EMI. In EMI/EMC applications where the victim is located outside the near-field zone of the radiator, the reactive power is of little concern. In packages, interconnects are easily exposed to the near field of a radiating source. Therefore, the interfering fields can be detrimental to the functionality of the system.

The total reactive power stored inside the structures are calculated by [18]

$$
P=\frac{\omega}{2} \int_{V}\left(\mu^{\prime}|\vec{H}|^{2}-\varepsilon^{\prime}|\vec{E}|^{2}\right) d v .
$$

The first term in the integrand defines the magnetic power density and the second term defines the electric power density. If the result of integral at the frequency of interest is positive, the stored magnetic power is greater than stored electric power and vice versa.

In the EBG-patterned parallel-plate setup, both propagating and evanescent modes create reactive power. For propagating modes, the reactive power is stored throughout the entire structures. For an evanescent mode, the field is mainly localized around the active port. The field inside the setup decays with distance. As a result, the reactive power of evanescent mode is densely stored close to the active port. This is demonstrated by the reactive power distributions shown in Figures 13(a)-13(c). These figures, respectively, present the net reactive power densities stored in the setup for $2 \mathrm{GHz}$, where the mode propagates, for $3.5 \mathrm{GHz}$, where the mode is evanescent, and for $4.5 \mathrm{GHz}$, where the higherorder mode is evanescent. The dark red color in the color scale map represents the largest density value of net reactive positive power which is magnetic in nature. Similarly, the dark blue color corresponds to largest density value of net reactive negative power which is electric in nature. The green color at the middle of map corresponds to approximately zero where the magnetic power and electric power are practically equal.

From the opening of EBG structures, the reactive power partially radiates to the surrounding environment. Hence, near-fields carrying both real and reactive powers exist close to the EBG structures. The reactive power decays very fast and the fields delivering only real power reach the far zone. The near-fields similar to far-fields can couple energy to any components located in their interference zone. This coupling is based on both real and reactive power. Figure 14 shows four different lines located very close to the EBG-patterned parallel-plane setup. These lines, which are labeled as Line 1, Line 2, Line 3, and Line 4, are used to assess the fields created by the radiated power from the setup. The fields are numerically computed via full-wave solver at these lines.

Figures 15(a) and 15(b) show the plots of the $E$-fields along Line 1 , for $1.5 \mathrm{GHz}, 2 \mathrm{GHz}, 2.5 \mathrm{GHz}, 2.9 \mathrm{GHz}, 3.5 \mathrm{GHz}$, and $4.5 \mathrm{GHz}$ (the fields are divided to two sets for readability). For all these frequencies, Line 1 is in the near-field zone of the radiating setup. The approximate boundary of the near field is defined by $R_{1}=0.62 \sqrt{D^{3} / \lambda}$ [20] and the approximate boundary of the far field is defined by $R_{2}=2 D^{2} / \lambda[20]$. $D$ is the largest dimension of EBG-board which is the distance represented by its diagonal line $(D=13.32 \mathrm{~mm}) . \lambda$ is the wavelength in the surrounding environment which in our case is free space. For example, at $3.5 \mathrm{GHz}, R_{1}=3.26 \mathrm{~mm}$ and $R_{2}=4.14 \mathrm{~mm}$. The active port is Port 2 and Port 1 acts as a receiver.

The local peaks in the field diagrams show that the fields are stronger at the openings of the EBG patterns and the board edges. The locations of openings and ports along the lines in the normalized scale are labeled in Figure 15(a). The strengths of the fields along the lines and the level of radiated powers change with frequency. The maximum peak of the field is not always close to the active port. At a frequency 


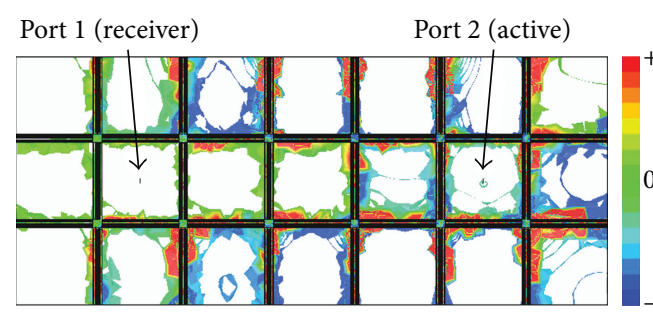

(a)

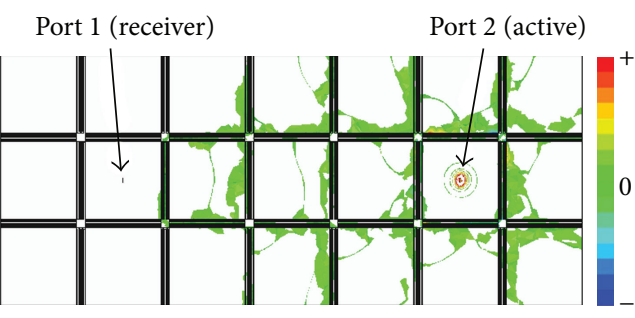

(b)

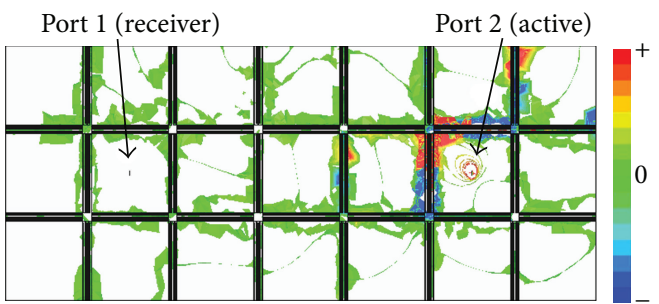

(c)

FIGURE 13: Reactive power density per volume stored inside the EBG-patterned parallel-plane setup: (a) at $2 \mathrm{GHz}$, (b) at $3.5 \mathrm{GHz}$, and (c) at $4.5 \mathrm{GHz}$. The color scale map shows the positive and negative range of power.

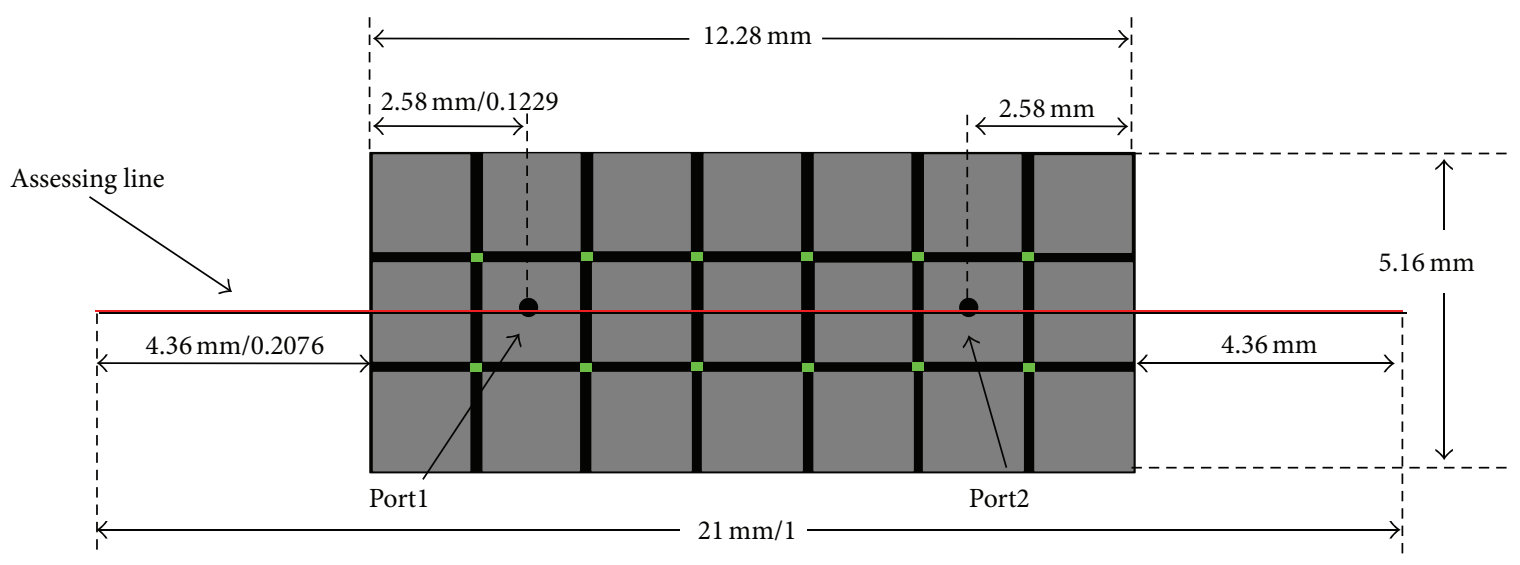

(a)

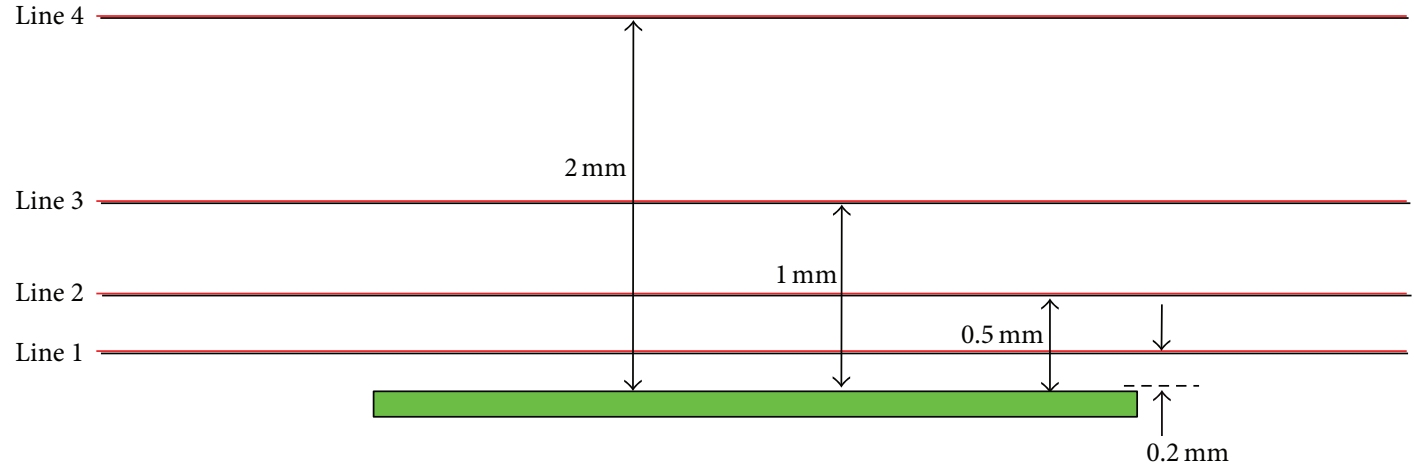

(b)

FIGURE 14: (a) Top view and (b) the side view of the EBG-patterned parallel planes and four assessing lines used to compute fields in the near zone. The dimensions are labeled in the figures. The distances along the assessing lines are shown in metric system and normalized form. 


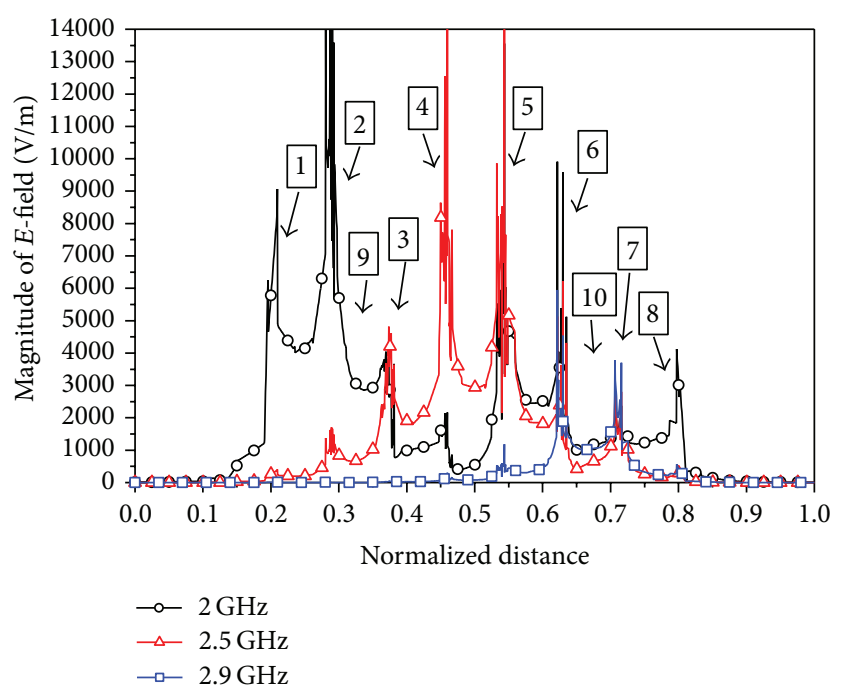

(a)

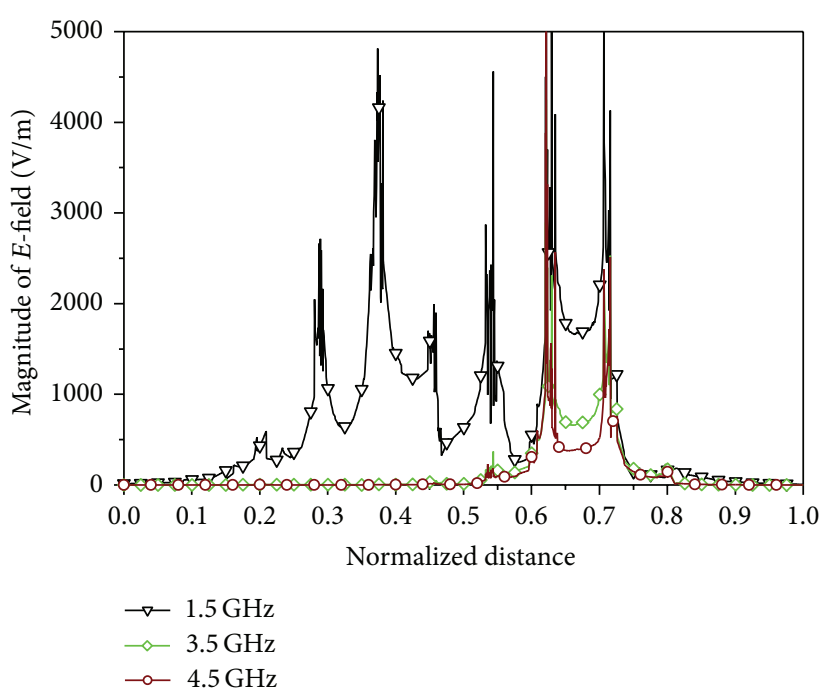

(b)

Figure 15: (a) and (b) Total E-fields along the assessing Line 1 at different frequencies. Line 1 shown in Figure 14 is distant from the EBGpatterned parallel planes by $0.2 \mathrm{~mm}$. In the normalized scale, Label 1 shows the left edge opening, label 8 shows the right edge opening, and labels 2-7 show the 1st to 6th meandered-pattern openings, respectively. Labels 9 and 10 show the locations of Port 1 and Port 2, respectively.

corresponding to a propagating mode (inside the board) such as $1.5 \mathrm{GHz}, 2 \mathrm{GHz}$, and $2.5 \mathrm{GHz}$, power radiates from all the openings of board. For a frequency that lies in the suppression band, which includes bandgaps and frequency regions corresponding to evanescent higher-order modes, such as $2.9 \mathrm{GHz}, 3.5 \mathrm{GHz}$, and $4.5 \mathrm{GHz}$, the fields and power are only localized around the active port inside the board. Therefore, the power mainly radiates out from the openings of board which are very close to the active port. Hence, the $E$-fields at Line 1 are higher close to the active port and they decay very fast with distance.

Figure 16 shows the $E$-fields at $2 \mathrm{GHz}$ along Line 1, Line 2, Line 3, and Line 4. For this frequency, all lines fall in the near-field zone of the EBG-patterned board. The $2 \mathrm{GHz}$ corresponds to a mode that propagates within the board; therefore, the radiated fields are detected all along the observation lines (Line 1 to Line 4). The fields are strongest close to the EBGs; however, they dramatically decay with distance. This confirms that the strong fields are mainly based on the reactive radiated powers. The fields reaching to the far zone are only delivering real power which in our case is very small.

These results clearly show that, in any package, the layers adjacent to the EBG patterns are exposed to strong emissions. Strong fields with reactive power can couple energy to adjacent components or interconnects. Therefore, these radiations can be an important source of EMI.

\section{Conclusion}

Planar EBG patterning of the package or board layer reduces unwanted waves propagating through these structures in the effective suppression band, thus, significantly minimizing the potential for switching noise. The suppression band

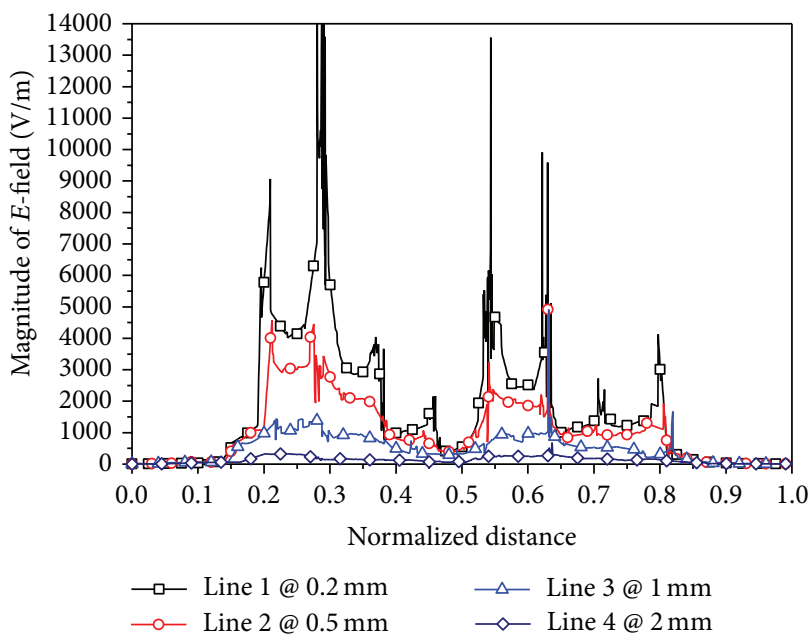

Figure 16: Total $E$-fields in near-field zone along four different assessing lines. The lines are shown in Figure 14.

which is derived from the transfer of energy between two locations within the board does not necessarily correspond to the bandgap obtained from the dispersion diagram. In fact, through the example considered in this study, the suppression band of EBG structures could possibly include several bandgaps as well as evanescent higher-order modes.

Planar EBGs in their suppression band are generally effective in suppressing the total power-loss peaks compared to the case when the planes are solid. Over the bandgap regions, the total power loss is negligible. However, there are significant total power losses corresponding to evanescent higher-order modes. Outside the effective suppression band, corresponding to propagating mode frequency regions, the total power losses are also substantial. For the planar EBGs 
based on high- $k$ materials, considerable amount of power loss takes place within the host medium. Very small portion of real power radiates to the neighboring environment. As a result, these EBG structures are highly effective for reducing switching noise and any consequential EMI. The radiated reactive powers at the near field zone of EBG structures, however, can be strong within and outside of the suppression band. The EMI through these fields can be very destructive if the frequencies of these fields happen to fall within clock frequencies and subsequent harmonics.

The near-fields are stronger close to the openings in EBG patterns and at the edges of the board or package. The strengths of these fields vary as functions of frequency and location. Also, the near-fields are detectable over the entire frequency range, including propagating- and evanescentmode frequency regions. Over the frequency range where modes can propagate, the fields exist along the structures and they decay fast with distance. For frequencies where the modes are evanescent, the fields are strongest close to the active port and decay very fast with distance. Hence, if the EBG patterning of a package results in an optimally wide effective suppression band where several higher-order modes are evanescent, then the total near-field emissions can be reduced significantly.

\section{Conflict of Interests}

There is no conflict of interests regarding the publication of this article.

\section{References}

[1] J. Müller and D. Josip, "Integrated capacitor using LTCC," in Proceedings of the Microtech Conference, Manchester, UK, January 2002.

[2] B. Mohajer-Iravani and O. M. Ramahi, "Miniaturized wideband plannar electromagnetic bandgap structures using high- $k$ dielectrics," in Proceedings of the IEEE Antennas and Propagation Society International Symposium, pp. 2921-2924, Honolulu, Hawaii, USA, June 2007.

[3] B. Mohajer-Iravani and O. M. Ramahi, "EMI suppression in microprocessor packages using miniaturized electromagnetic bandgap structures with high-k dielectrics," in Proceedings of the IEEE International Symposium on Electromagnetic Compatibility, pp. 1-4, Honolulu, Hawaii, USA, July 2007.

[4] B. Mohajer-Iravani and O. M. Ramahi, "Suppression of EMI and electromagnetic noise in packages using embedded capacitance and miniaturized electromagnetic bandgap structures with high-k dielectrics," IEEE Transactions on Advanced Packaging, vol. 30, no. 4, pp. 776-788, 2007.

[5] B. Mohajer-Iravani and O. M. Ramahi, "Design of EBG structures based on wideband circuit model," IEEE Transactions on Advanced Packaging, vol. 33, no. 1, pp. 169-179, 2010.

[6] B. Mohajer-Iravani and O. M. Ramahi, "Radiating emissions from the planar electromagnetic bandgap (EBG) structures," in Proceedings of the IEEE International Symposium on Electromagnetic Compatibility, pp. 780-783, Fort Lauderdale, Fla, USA, July 2010.

[7] J. Qin, O. M. Ramahi, and V. Granatstein, "Novel planar electromagnetic bandgap structures for mitigation of switching noise and EMI reduction in high-speed circuits," IEEE Transactions on Electromagnetic Compatibility, vol. 49, no. 3, pp. 661-669, 2007.

[8] O. M. Ramahi, V. Subramanian, and B. Archambeault, "Simple and efficient finite-difference frequency-domain algorithm for study and analysis of power plane resonance and simultaneous switching noise in printed circuit boards and chip packages," IEEE Transactions on Advanced Packaging, vol. 26, no. 2, pp. 191198, 2003.

[9] J. Choi, D. G. Kam, D. Chung et al., "Near-field and far-field analyses of alternating impedance electromagnetic bandgap (AI-EBG) structure for mixed-signal applications," IEEE Transactions on Advanced Packaging, vol. 30, no. 2, pp. 180-190, 2007.

[10] Y.-W. Huang, T.-K. Wang, and T.-L. Wu, "Design and modelling of miniaturized bandgap structure for wideband $\mathrm{GHz}$-noise suppression based on LTCC technology," IEEE Transactions on Advanced Packaging, vol. 33, no. 3, pp. 630-638, 2010.

[11] T.-L. Wu, H.-H. Chuang, and T.-K. Wang, "Overview of power integrity solutions on package and PCB: decoupling and EBG isolation," IEEE Transactions on Electromagnetic Compatibility, vol. 52, no. 2, pp. 346-356, 2010.

[12] T.-L. Wu, Y.-H. Lin, T.-K. Wang, C.-C. Wang, and S.-T. Chen, "Electromagnetic bandgap power/ground planes for wideband suppression of ground bounce noise and radiated emission in high-speed circuits," IEEE Transactions on Microwave Theory and Techniques, vol. 53, no. 9, pp. 2935-2942, 2005.

[13] B. Mohajer-Iravani, S. Shahparnia, and O. M. Ramahi, "Coupling reduction in enclosures and cavities using electromagnetic band gap structures," IEEE Transactions on Electromagnetic Compatibility, vol. 48, no. 2, pp. 292-303, 2006.

[14] R. Remski, "Analysis of photonic bandgap surfaces using Ansoft HFSS," Microwave Journal, vol. 43, no. 9, pp. 190-198, 2000.

[15] Ansoft Corporation, "High Frequency Structure Simulator (HFSS)," Pittsburgh, Pa, USA.

[16] L. Brillouin, Wave Propagation in Periodic Structures: Electric Filters and Crystal Lattices, McGraw-Hill, New York, NY, USA, 1946.

[17] B. Mohajer-Iravani, Electromagnetic interference reduction using electromagnetic bandgap structures in packages, enclosures, cavities, and antennas [Ph.D. dissertation], Department of Electrical and Computer Engineering, University of Maryland, College Park, Md, USA, 2007.

[18] D. M. Pozar, Microwave Engineering, Addison-Wesley, Reading, Mass, USA, 1990.

[19] B. Mohajer-Iravani and O. M. Ramahi, "Reactive power radiated from the planar electromagnetic bandgap structures, a source of EMI in high speed packages," in Proceedings of the IEEE International Symposium on Antennas and Propagation and USNC/URSI National Radio Science Meeting, pp. 1840-1843, Spokane, Wash, USA, July 2011.

[20] C. A. Balanis, Antenna Theory Analysis and Design, John Wiley \& Sons, New York, NY, USA, 1997. 

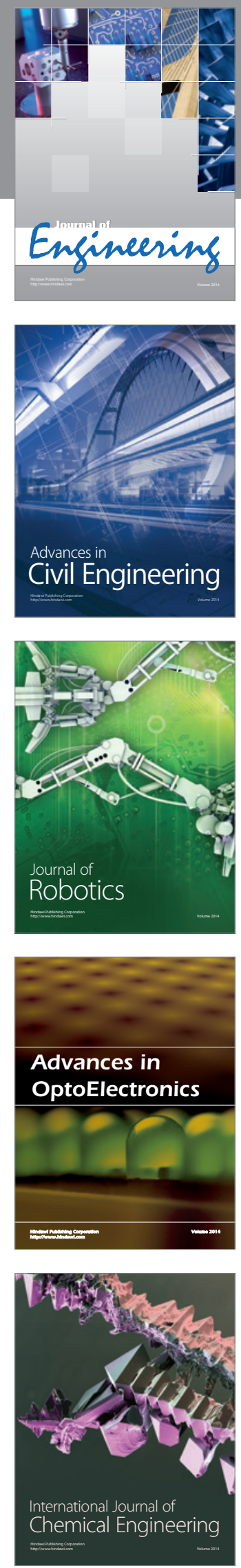

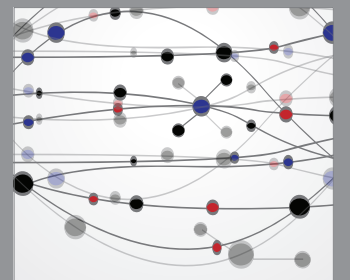

The Scientific World Journal
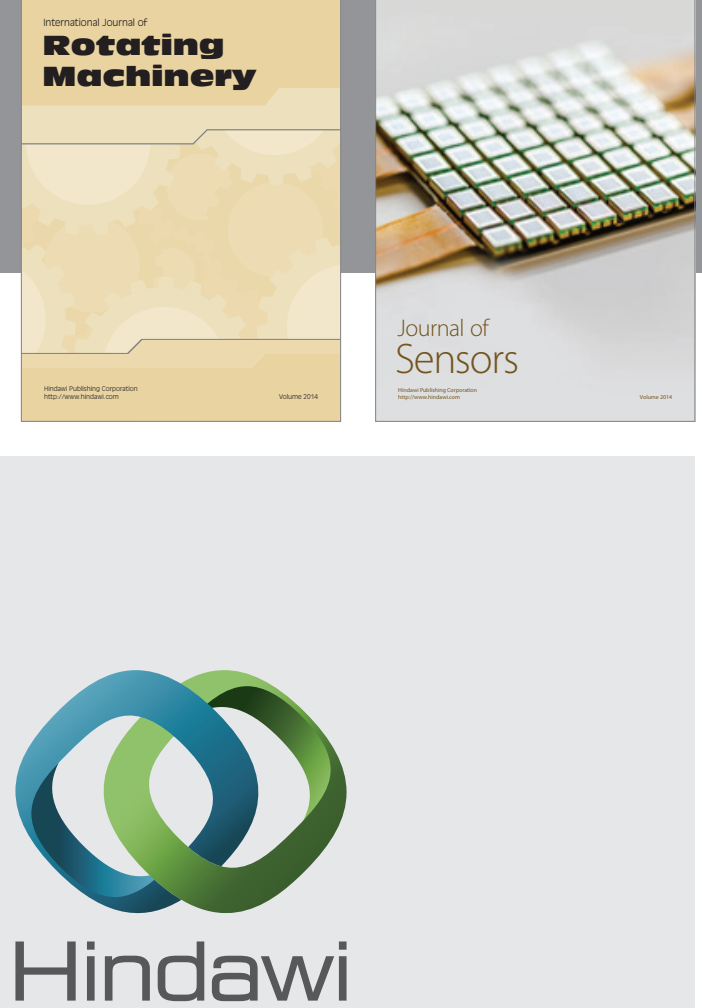

Submit your manuscripts at http://www.hindawi.com
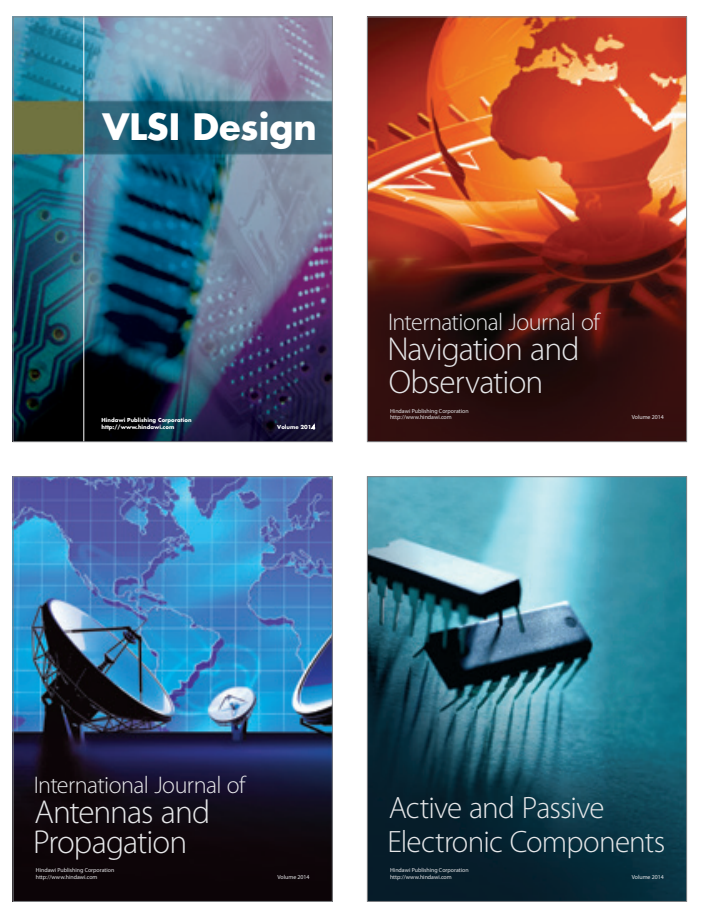
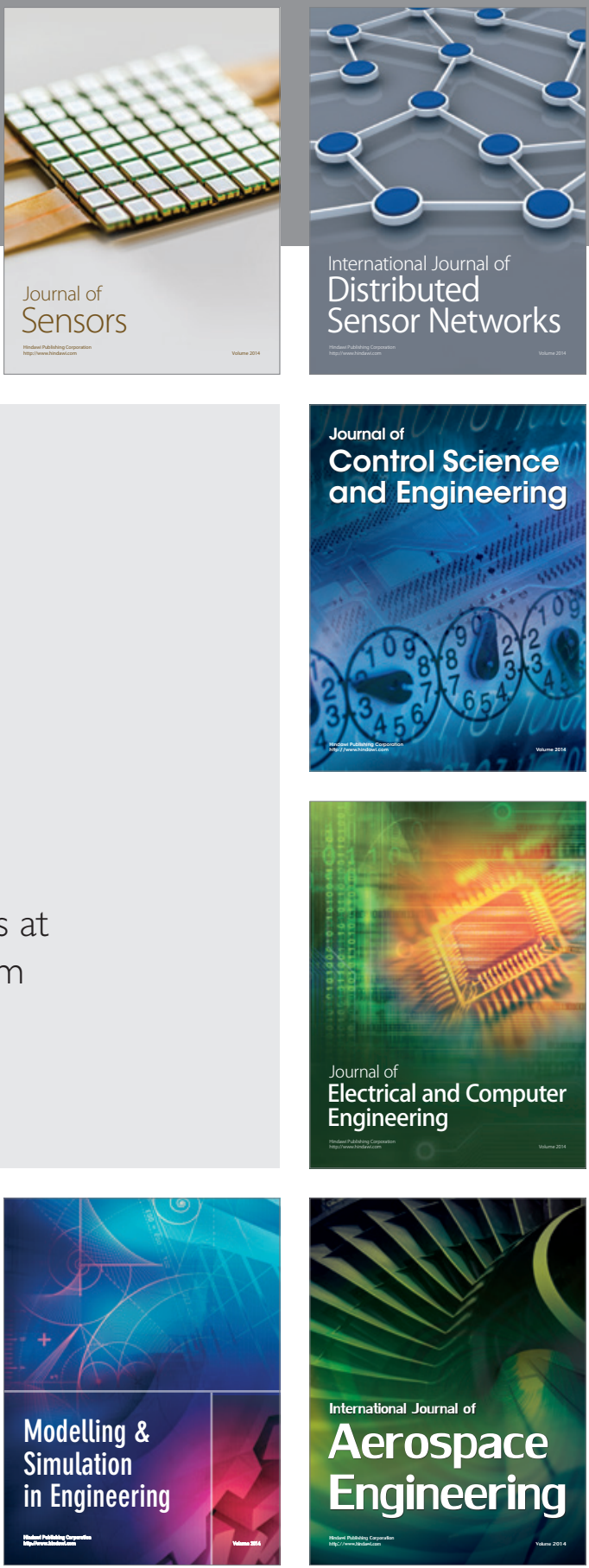

Journal of

Control Science

and Engineering
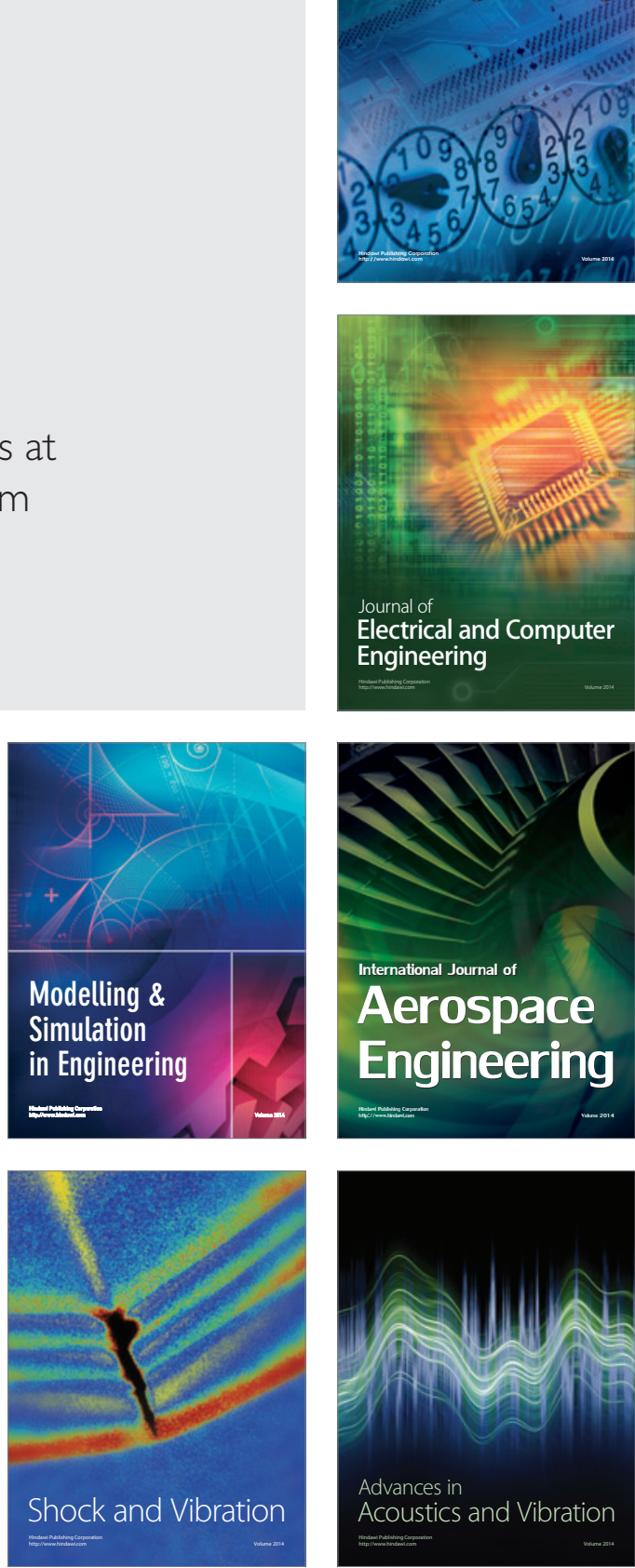